\title{
Du VIH/sida aux tumeurs malignes associées
}

\section{From HIV/AIDS to associated malignancies}

\author{
A. $\mathbf{L y}$ \\ (C) Springer-Verlag 2009
}

L'induction d'une déficience de l'immunité de l'hôte, la variabilité génétique et l'intégration au génome cellulaire sont quelques-unes des redoutables armes qui permettent au virus du syndrome d'immunodéficience acquise (sida) d'échapper à la défense de l'organisme, de se répliquer et de se transmettre si efficacement que cette pathologie virale est devenue en peu d'années une des pandémies les plus marquantes de l'histoire de l'épidémiologie. Ces mêmes propriétés ont empêché jusqu'ici l'élaboration d'un vaccin prophylactique véritablement protecteur et sont également à la base de la formidable chimiorésistance du virus de l'immunodéficience humaine (VIH) [1].

L'expansion autant épidémiologique que géographique de ce rétrovirus est responsable de la mort de 25 millions de personnes depuis 1983, date de sa découverte par une équipe de l'Institut Pasteur de Paris [2]. Environ 65 millions d'individus ont été infectés par le VIH au cours des dernières décennies [1]. L'incidence du VIH/sida est estimée à 2,7 millions de personnes et sa mortalité à deux millions en 2007 [1]. Sa prévalence actuelle est de 33 millions de patients dont la moitié sont des femmes et 1,9 million des enfants de moins de 15 ans [1]. Cependant, grâce aux campagnes de prévention, un ralentissement de la dynamique de la maladie est observé, quoique 7300 nouvelles contaminations surviennent chaque jour dans le monde [1].

Si toutes les aires géographiques sont impactées par le VIH, la pandémie du sida sévit de façon disproportionnée à l'échelle mondiale. L'Afrique subsaharienne reste le continent le plus durement frappé par cette maladie qui pourrait, selon certains postulats, trouver ses origines sur ces mêmes terres, même si le premier cas de sida a été découvert aux États-Unis en 1981 [1,3-5].

L'évolution du virus de l'immunodéficience simienne (VIS) infectant les singes mangabey vivant dans les régions Ouest-africaines serait l'explication du mystère associé à l'émergence subite de cette pathologie. Le VIS a de grandes similitudes de séquences avec le VIH-2 [3,6,7]. Des séries

A. Ly $(\bowtie)$

Afrocancer, BP 60751, F-75827 Paris cedex 17, France

e-mail : adamaly@gmail.com de mutations génétiques pourraient rendre possible le passage du virus VIS du singe à l'homme abolissant la barrière d'espèce et fournissant un avantage sélectif à une adaptation humaine $[3,4]$.

De même, des chimpanzés d'Afrique équatoriale constituent un réservoir pour un virus, le VIS-CPZ, un variant proche du VIH-1 [3,4]. Le VIH-1 partage avec le VIH-2 environ 55 à $60 \%$ d'homologie de séquences en aminoacides si l'on considère les protéines les plus conservées (gag et pol) [5]. Ce sont ces données qui ont fondé, en partie, les arguments les plus plausibles sur l'histoire originelle du sida. Toutefois, il y a absence de consensus sur ces analyses et interprétations épidémiologiques. D'autres modèles explicatifs à la fois de l'origine du VIH et de l'origine du sida existent [3-5,7-9].

Il reste qu'en Afrique subsaharienne, le sida est une cause majeure de mortalité ( $75 \%$ du total des décès dans le monde), et qu'on y dénombre deux tiers des personnes séropositives dans le monde, soit 22 millions de cas. La situation épidémiologique demeure préoccupante puisque près de deux millions de personnes nouvellement infectées y ont été enregistrées en 2007 selon le rapport du Programme mondial des Nations Unies sur le VIH/sida (Onusida) [1].

L'avènement des thérapies antirétrovirales (mono-, bi-, trithérapies) a suscité un grand espoir chez les malades et pour la lutte contre le sida en général.

Incontestablement, ces nouvelles molécules et leurs combinaisons ont permis sinon une guérison définitive du moins d'allonger l'espérance de vie des sidéens. En effet, en dépit des contraintes de prises régulières de plusieurs médicaments et des manifestations secondaires indésirables, l'accès aux traitements antirétroviraux fait que les patients vivent plus longtemps avec la maladie [1,3,10]. En Afrique subsaharienne, une infime minorité des personnes infectées par le VIH peut disposer de ces thérapies antirétrovirales [1,11-13]. Malgré le programme mondial « trois millions d'ici $2005 »$ de l'Onusida et de l'Organisation mondiale de la santé (OMS), la couverture de la thérapie antirétrovirale n'est passée que de 2 à $17 \%$ dans cette région, alors que 4,7 millions de malades doivent recourir à ces traitements [14]. 
Ainsi, une des principales avancées de la lutte contre le $\mathrm{VIH} /$ sida a donc été le passage d'une pathologie pouvant être rapidement mortelle à une maladie chronique avec laquelle il est de plus en plus possible de vivre avec une qualité de vie qui ne cesse de s'améliorer. La chronicité de la maladie est le reflet d'un acquis considérable en attendant des alternatives vaccinales ou thérapeutiques et une meilleure prévention de la propagation du virus.

Seulement, une des conséquences de l'évolution clinique de cette maladie virale est l'installation d'une immunodéficience potentiellement sévère et qui expose à de multiples infections dites opportunistes (tuberculose, pneumocystose, candidose, etc.) $[1,13,15]$.

$\mathrm{Au}$ plan physiopathologique, cette dégradation progressive et parfois inexorable de l'état de santé général induit par le VIH est corrélée à la baisse de l'efficacité du système immunitaire. Cette perte d'immunocompétence est ellemême liée à la diminution du nombre de lymphocytes $\mathrm{T}$ CD4+ et de macrophages qui expriment aussi ce marqueur (CD4) à la surface de leur membrane plasmique. Le deuxième marqueur exprimé par ces cellules et qui est indispensable à leur infectivité est une chimiokine, le CCR5 [16]. Les souches virales - VIH-1 et VIH-2 - ont une forte affinité pour ces catégories cellulaires qui jouent des fonctions déterminantes dans l'immunorégulation, qu'elle soit humorale (TH2) ou à médiation cellulaire (TH1). La réplication du VIH dans les cellules de l'immunité génère les effets cytopathogènes, les déplétions cellulaires et les phénomènes apoptotiques observés au cours de l'infection $[3,17]$.

Par ailleurs, l'autre versant de cette déficience acquise du système immunitaire est la genèse de tumeurs cancéreuses [11,18-20]. Cette altération de la réponse immune expose 30 à $40 \%$ des personnes infectées par le VIH à un risque accru de développer un cancer [21]. En effet, les données épidémiologiques montrent que, par rapport à la population générale, la fréquence de certains types néoplasiques est augmentée chez ces sujets [20-23].

Ces tumeurs malignes associées au VIH/sida sont le sarcome de Kaposi, les lymphomes malins non hodgkiniens (LMNH), le cancer invasif du col de l'utérus, la maladie de Hodgkin, les cancers anogénitaux [18,23-25].

Quant au rôle du VIH dans le processus de tumorisation, il est indirect. Le VIH n'est pas un virus oncogène. Ce sont les perturbations immunologiques et les actions immunosuppressives qu'il entraîne qui sont aggravantes et favorisent l'émergence de tumeurs malignes. Alors, il est classé par le Centre international de recherche sur le cancer (CIRC) en catégorie I [26].

On remarquera que la caractéristique commune à ces néoplasies associées à l'infection par le VIH est, d'un point de vue étiologique, causée par des virus oncogènes comme le virus d'Epstein-Barr, l'herpèsvirus humain de type 8 (encore appelé virus du sarcome de Kaposi) ou le virus du papillome humain.

En Afrique, la progression des cancers classant sida a été considérable.

Le sarcome de Kaposi est devenu le cancer le plus fréquent et le plus mortel chez l'homme [20]. Le cancer du col utérin est en pôle position dans le classement des cancers chez la femme [20]. Les LMNH sont 200 fois plus fréquents chez les malades du sida que chez les personnes non infectées [20,21].

L'explosion des cas de sida est directement à l'origine de la fulgurance de l'augmentation des pathologies tumorales sur le continent africain. Les estimations qui sont de 667000 nouveaux cas en 2008 passeront, d'ici à 2020, à un million de nouveaux cas chaque année [20].

En tout état de cause, l'élargissement de l'accès aux traitements antirétroviraux innovants au plus grand nombre des pays en voie de développement est inéluctable si on veut y contrecarrer la pandémie du sida. Dans un tel schéma, changeront le pronostic vital et la longévité des patients africains. Le corollaire sera un accroissement de l'incidence des affections malignes. Ce qui constitue un double défi à relever. Mais, dans les pays du Nord, les thérapeutiques antirétrovirales hautement actives (highly active antiretrovial therapies [HAART]) ont permis de réduire la morbidité associée aux infections opportunistes et l'incidence de certains cancers associés au VIH/sida tels le sarcome de Kaposi ou les LMNH dans une moindre mesure [14,18,22,24].

En revanche, les résultats sont décevants pour le cancer invasif du col utérin, la maladie de Hodgkin et les cancers anogénitaux [22,27].

Face à ces infections multiples, l'offre thérapeutique doit, à l'évidence, être adaptée à la nouvelle donne sanitaire et améliorée à la fois qualitativement et quantitativement. De surcroît, elle devra être marquée du sceau de la pluridisciplinarité.

$\mathrm{Au}$ niveau fondamental, ces co-infections virales ont fourni un vaste champ d'expérimentation au concept d'immunosurveillance des cancers, formulé par Burnet en 1957, selon lequel le système immunitaire reconnaît et détruit continûment les cellules transformées avant même qu'elles acquièrent une taille détectable pouvant menacer l'homéostasie de l'organisme $[28,29]$.

La faillite de ce rôle du système immunitaire dans le contrôle de la prolifération tumorale en cas d'immunodéficience acquise ou congénitale et lors de traitements immunosuppressifs explique le développement de certains cancers normalement jugulés par le système immunitaire.

Pourtant, d'autres formes néoplasiques surviennent malgré cette immunosurveillance et au cours d'une immunosuppression. Ce sont notamment les cancers du sein, du poumon, du foie, de la prostate et du côlon rectum 
qui sont particulièrement prévalents dans la population générale [20,22,24].

Pour ces cancers non associés à l'infection par le VIH/ sida, ce sont plutôt les facteurs de risque classiques qui rendent compte de leur genèse [18,27].

D'ailleurs, la disponibilité des thérapies antirétrovirales hautement actives n'a pas permis leur diminution de façon significative chez les sujets infectés par le VIH [22,27].

À la lumière des récentes découvertes, ce concept d'immunosurveillance des cancers a évolué et a été remplacé par celui d'immunoediting. Ce nouveau concept tout en englobant le premier propose deux phases supplémentaires pour expliquer l'immunité antitumorale : une phase d'équilibre durant laquelle les cellules tumorales peu immunogènes et non éliminées par le système immunitaire survivent longtemps sans prolifération excessive. Il y a une forme de sélection et de «tolérance » des cellules cancéreuses les plus résistantes.

La dernière phase, celle de l'échappement, est la rupture de cet équilibre qui autorise une croissance tumorale libre et illimitée [30-32].

Ainsi, une meilleure compréhension de ces interactions entre le système immunitaire et les tissus tumoraux permettra des avancées sur la connaissance des bases fondamentales de la cancérogenèse et ouvrira de nouvelles perspectives d'immunothérapie anticancéreuse.

Au total, tout ce qui est entrepris en termes de prévention, de traitements et de financement pour le recul du sida est soluble dans le combat contre le cancer qui, dans cette thématique, est l'étape d'après pour un nombre croissant de malades. Inversement, de la prévention aux diagnostics, des thérapies aux soins palliatifs, les divers aspects de la lutte antitumorale impliquent les malades du VIH/sida et croisent leur réalité quotidienne puisqu'ils ont un risque de survenue de tumeurs malignes qui s'accroît à mesure que l'immunodéficience dont ils souffrent s'aggrave. En conséquence, la somme des efforts consentis dans ces deux parties d'un même combat contribue au renversement des perspectives épidémiologiques de ces pathologies sur le continent africain et à l'amélioration de la santé des populations.

À cet égard, le cloisonnement des disciplines motivé par des logiques corporatistes est une impasse. Il risquerait d'occulter la question essentielle du sens de l'action médicale et de l'importance des activités de recherche biomédicale. Les synergies, dès lors évidentes, qui doivent fournir des réponses globales et objectives tant ces enjeux de santé et leurs implications sociales et économiques, sont énormes.

\section{Références}

1. Onusida (2006) Rapport sur l'épidémie mondiale de sida : résumé d'orientation : Programme mondial des Nations Unies sur le VIH/sida (Onusida). Onusida/06.20F
2. Barré-Sinoussi F, Chermann JC, Rey F, et al (1983) Isolation of a Tlymphotropic retrovirus from a patient at risk for acquired immune deficiency syndrome (AIDS). Science. 220(4599): 868-71

3. Montagnier L (1994) Des virus et des hommes, Éditions Odile Jacob, 315p

4. Karpas A (2004) Human retroviruses in leukaemia and AIDS: reflections on their discovery, biology and epidemiology. Biol Rev Camb Philos Soc 79(4):911-33

5. Peeters M, Piot P, Van der Groen G (1991) Variability among HIV and SIV strains of African origin. AIDS 5(Suppl 1):S29-S36

6. Apetrei C, Robertson DL, Marx PA (2004) The history of SIVS and AIDS: epidemiology, phylogeny and biology of isolates from naturally SIV infected non-human primates (NHP). Afr Front Biosci 9:225-54

7. Marx PA, Apetrei C, Drucker E (2004) AIDS as a zoonosis? Confusion over the origin of the virus and the origin of the epidemics. J Med Primatol 33(5-6):220-6

8. Pandrea I, Silvestri G, Apetrei C (2009) AIDS in African nonhuman primate hosts of SIVs: a new paradigm of SIV infection. Curr HIV Res 7(1):57-72

9. Alizon M, Montagnier L (1987) Genetic variability in human immunodeficiency viruses. Ann NY Acad Sci 511:376-84

10. Grulich AE (2009) Living longer with HIV: what does it mean for cancer risk? Curr Opin HIV AIDS 4(1):1-2

11. Mbulaiteye SM, Parkin DM, Rabkin CS (2003) Epidemiology of AIDS-related malignancies an international perspective. Hematol Oncol Clin North Am 17(3):673-96

12. Yakam JC, Gruénais ME (2009) Involving new actors to achieve ART scaling-up: difficulties in an HIV/AIDS counselling and testing centre in Cameroon. Int Nurs Rev 56(1):50-7

13. Yaotsè DA, Nicole V, Roch NF, et al (2009) Genetic characterization of HIV-1 strains in Togo reveals a high genetic complexity and genotypic drug-resistance mutations in ARV naive patients. Infect Genet Evol 9(4):646-52

14. Onusida/OMS (2006) Élargissement de l'accès au traitement antirétroviral dans le monde : rapport sur l'initiative «trois millions d'ici 2005 » et au-delà, 2006, OMS

15. Maher D, Harries A, Getahun H (2009) Tuberculosis and HIV interaction in Sub-Saharan Africa: impact on patients and programmes; implications for policies. Trop Med Int Health 10(8): 734-42

16. Lieberman-Blum SS, Fung HB, Bandres JC (2008) Maraviroc: a CCR5-receptor antagonist for the treatment of HIV-1 infection. Clin Ther 30(7):1228-50

17. Nies-Kraske E, Schacker TW, et al (2009) Evaluation of the pathogenesis of decreasing $\mathrm{CD} 4(+) \mathrm{T}$ cell counts in human immunodeficiency virus type 1 -infected patients receiving successfully suppressive antiretroviral therapy. J Infect Dis 199(11):1648-56

18. Engels EA, Biggar RJ, Hall HI, et al (2008) Cancer risk in people infected with human immunodeficiency virus in the United States. Int J Cancer 123(1):187-94

19. Mbulaiteye SM, Katabira ET, Wabinga H, et al (2006) Spectrum of cancers among HIV-infected persons in Africa: the Uganda AIDS-Cancer Registry Match Study. Int J Cancer 118(4): 985-90

20. Centre international de recherche sur le cancer (2008) Le cancer dans le monde en 2008. Centre international de recherche sur le cancer, Lyon

21. Centre international de recherche sur le cancer (2005) Le cancer dans le monde. Centre international de recherche sur le cancer, Lyon

22. Crum-Cianflone N, Hullsiek KH, Marconi V, et al (2009) Trends in the incidence of cancers among HIV-infected persons and the impact of antiretroviral therapy: a 20 -year cohort study. AIDS 23(1):41-50 
23. Weiss R (2006) Les tumeurs malignes associées au VIH/sida In: Ly A, Khayat D (eds) Le cancer en Afrique : de l'épidémiologie aux applications et perspectives de la recherche biomédicale. Institut national du cancer (INCa), Paris, pp. $479-89$

24. Silverberg MJ, Abrams DI (2007) AIDS-defining and nonAIDS-defining malignancies: cancer occurrence in the antiretroviral therapy era. Curr Opin Oncol 19(5):446-51

25. Kahesa C, Mwaiselage J, Wabinga HR, et al (2008) Association between invasive cancer of the cervix and HIV-1 infection in Tanzania: the need for dual screening. BMC Public Health 8:26

26. IARC (1996) Monographs on the evaluation of carcinogenic risks to humans, vol 67 human immunodeficiency viruses and human T-cell lymphotropic viruses. Consultable http://monographs.iarc.fr/ENG/Monographs/vol67/mono67-5.pdf
27. Silverberg MJ, Abrams DI (2009) Do antiretrovirals reduce the risk of non-AIDS-defining malignancies? Curr Opin HIV AIDS 4(1):42-51

28. Burnet M (1957) Cancer: a biological approach. III. Viruses associated with neoplastic conditions. IV. Practical applications. Br Med J 1:841-7

29. Burnet FM (1970) The concept of immunological surveillance. Prog Exp Tumor Res 13: 1-27

30. Dunn GP, Old LJ, Schreiber RD (2004) The three Es of cancer immunoediting. Annu Rev Immunol 22:329-60

31. Teng MW, Swann JB, Koebel CM, et al (2008) Immune-mediated dormancy: equilibrium with cancer. J Leukoc Biol 84(4):988-93

32. Reiman JM, Kmieciak M, Manjili MH, Knutson KL (2009) Tumor immunoediting and immunosculpting pathways to cancer progression. Semin Cancer Biol 17(4):275-87 